DOI: 10.1002/adsc.201((will be filled in by the editorial staff))

\title{
Organocatalytic Enantioselective Friedel-Crafts Alkylation of 1- Naphthol Derivatives and Activated Phenols with Ethyl Trifluoropyruvate
}

\author{
Marc Montesinos-Magraner, ${ }^{\mathrm{a}}$ Carlos Vila, ${ }^{\mathrm{a}}$ Gonzalo Blay, ${ }^{\mathrm{a}}$ Isabel Fernández, ${ }^{\mathrm{a}} \mathrm{M}$. \\ Carmen Muñoz ${ }^{\mathrm{b}}$ and José R. Pedro ${ }^{\mathrm{a}, *}$ \\ a Departament de Química Orgànica, Facultat de Química, Universitat de València, Dr. Moliner 50, 46100 Burjassot, \\ València (Spain); \\ [phone: +0034963544329; e-mail: jose.r.pedro@uv.es] \\ b Departament de Física Aplicada, Universitat Politècnica de València, Camí de Vera s/n, 46022 València (Spain)
}

Received: ((will be filled in by the editorial staff))

Supporting information for this article is available on the WWW under http://dx.doi.org/10.1002/adsc.201\#\#\#\#\#\#.((Please delete if not appropriate))

\begin{abstract}
An organocatalytic enantioselective FriedelCrafts alkylation of a series of substituted 1-naphthol derivatives and activated phenols with ethyl trifluoropyruvate, catalyzed by a quinine-derived squaramide, is presented. Good yields and high to excellent enantioselectivities of the Friedel-Crafts alkylation products were obtained.
\end{abstract}

Keywords: asymmetric catalysis; Friedel-Crafts reaction; naphthols; organocatalysis; trifluoropyruvates

The Friedel-Crafts (F-C) reaction is one of the most fundamental, important and powerful $\mathrm{C}-\mathrm{C}$ bond forming reaction in organic chemistry. ${ }^{[1]}$ This reaction provides an efficient synthetic pathway to prepare functionalized aromatic compounds, of great importance and widely used in academia and industry. Moreover, the catalytic enantioselective version of the $\mathrm{F}-\mathrm{C}$ reaction leads to the formation of highly valuable chiral aromatic compounds. ${ }^{[2]}$

However, most of the examples reported in the literature with regard to this version are concerned to the use of heteroarenes such indoles and pyrroles as nucleophilic partners, ${ }^{[3]}$ whilst other nucleophiles such naphthols have been less studied, probably due to their reduced reactivity towards electrophiles. ${ }^{[4]}$ Consequently, the extension of the asymmetric F-C alkylations of naphthols is of great interest for organic synthesis. Since the first example of Erker in $1990^{[5]}$ where the enantioselective alkylation of naphthol was described using an activated ketone such methyl pyruvate, only two asymmetric examples of the reaction between naphthols and ketones have been recently reported, using isatins as substrates. ${ }^{[6]}$ Nevertheless, the enantioselective alkylation of 1naphthol with alkyl trifluoropyruvates is not described, to the best of our knowledge. This reaction would provide access to chiral tertiary benzylic alcohols bearing a trifluoromethyl group as a substituent. ${ }^{[7,8]}$ This particular motif has become an important structural characteristic in several drugs such as CJ17493 or Efavirenz (Figure 1). ${ }^{[9]}$

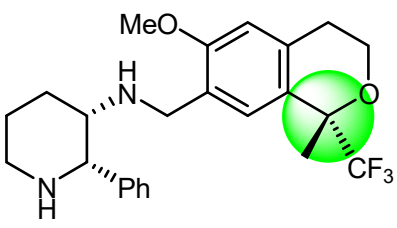

CJ-17493

(neurokinin 1 receptor antagonist)

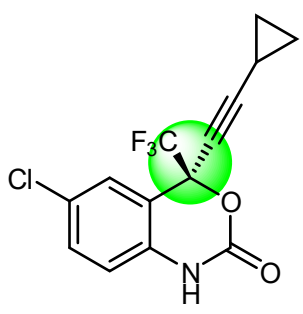

Efavirenz (anti-HIV)
Figure 1. Structures of drugs containing a chiral tetrasubstituted carbon bearing a trifluoromethyl group.

Since the pioneering work by Jørgensen and coworkers, ${ }^{[10]}$ who described the alkylation of different aromatic and heteroaromatic compounds with alkyl trifluoropyruvate, several methodologies have been reported ${ }^{[11]}$ However, the enantioselective alkylation of naphthols with ethyl trifluoropyruvate remains elusive (Scheme 1) ${ }^{[12]}$ As a part of our continuous interest in the enantioselective synthesis of $\mathrm{CF}_{3}$ cointaining compounds, ${ }^{[13]}$ herein we report an organocatalytic enantioselective F-C alkylation of 1naphthol derivatives with ethyl trifluoropyruvate under mild conditions using a squaramide organocatalyst ${ }^{[14]}$ derived from quinine. 


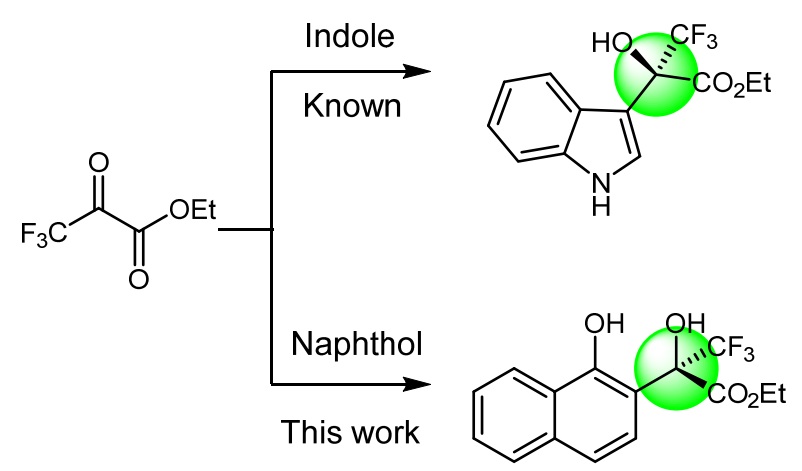

Scheme 1. Enantioselective Friedel-Crafts alkylation with ethyl trifluoropyruvate.

The reaction of 1-naphthol (1a) with ethyl trifluoropyruvate (2) was studied for different bifunctional organocatalyst under various reaction conditions (Table 1). We initially examined quinine (A) in toluene at rt (entry 1, Table 1). To our delight, the corresponding tertiary alcohol 3a was obtained smoothly in $70 \%$ yield and $43 \%$ ee. $9-O-(p \mathrm{Me}$ $\mathrm{C}_{6} \mathrm{H}_{4} \mathrm{CH}_{2}$ )-cupreine $\mathbf{B}$, where the $9-\mathrm{OH}$ group was protected and the 6'-OH was free, showed an inversion of the enantioselectivity $(-48 \%$ ee), with a moderate yield (Table 1, entry 2). Whereas, cupreine catalyst $\mathbf{C}$ and $\mathbf{D}$, with different groups such as aryl ether or benzoyl group at 9-OH position, afforded the product 3a with higher enantioselectivity, $(-78 \%$ ee, entries 3 and 4), but the reaction was slower. When quininederived thiourea $\mathbf{E}$ was used (Table 1, entry 5), the reaction proceed faster, obtaining the product in $86 \%$ yield, although with a decrease in the enantiomeric excess (-46\%). Quinine-derived squaramide $\mathbf{F}$ proved to be the most efficient catalyst (entry 6, Table 1) in terms of yield (89\%) and enantioselectivity (-78\% ee). Other thiourea organocatalyst bearing a tertiary amine moiety, such as Takemoto's catalyst G (entry 7, Table $1)$, shown good yield ( $86 \%$ yield), although with decreased selectivity $(46 \%$ ee). We decided to study the effect of different solvents (entries 8-12) with catalyst $\mathbf{F}$ in the Friedel-Crafts alkylation. To our delight, when ether was used as a solvent, excellent results were obtained ( $92 \%$ yield, 91\% ee, entry 9). The enantiomeric excess of tertiary alcohol 3a could reach $97 \%$ (entry 13) when the reaction was carried out at $0{ }^{\circ} \mathrm{C}$. Finally, the catalyst loading was decreased to 2 and $1 \mathrm{~mol} \%$ (entries 14 and 15, respectively), with similar results in terms of enantioselectivity, although the reactivity was much lower.

Table 1. Optimization of the reaction conditions. ${ }^{\text {a }}$

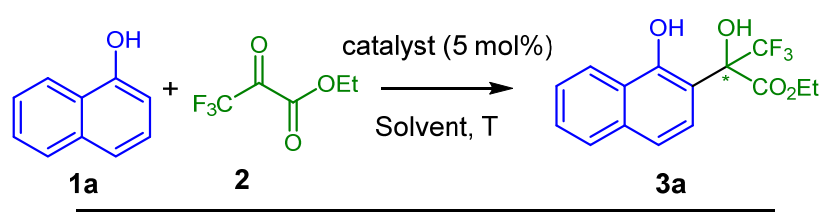<smiles>[R]NC(=S)N[C@H](c1ccnc2ccc(OC)cc12)N1C[C@@H]2CCN1C[C@@H]2C=C[C@H]1CN2C[C@H]1C[C@@H]2[C@H](O[R])c1ccnc2ccc(O)cc12</smiles>

A

B $\left(\mathrm{R}=4-\mathrm{MeC}_{6} \mathrm{H}_{4} \mathrm{CH}_{2}\right)$

$E\left(\mathrm{R}=3,5-\left(\mathrm{CF}_{3}\right)_{2}-\mathrm{C}_{6} \mathrm{H}_{3}\right)$

C $\left(\mathrm{R}=3,5-\mathrm{Me}_{2} \mathrm{C}_{6} \mathrm{H}_{3}\right)$<smiles>[R]Nc1c(NC(c2ccnc3ccc(OC)cc23)[C@H]2CC3CCN2C[C@@H]3C=C)c(=O)c1=O</smiles>

$F\left(\mathrm{R}=3,5-\left(\mathrm{CF}_{3}\right)_{2}-\mathrm{C}_{6} \mathrm{H}_{3}\right)$

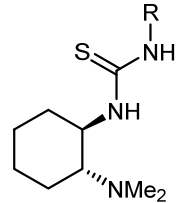

$\mathbf{G}\left(\mathrm{R}=3,5-\left(\mathrm{CF}_{3}\right)_{2}-\mathrm{C}_{6} \mathrm{H}_{3}\right)$

\begin{tabular}{|c|c|c|c|c|c|c|}
\hline Entry & $\begin{array}{l}\text { Catalyst } \\
(\mathrm{mol} \%)\end{array}$ & Solvent & $\mathrm{t}(\mathrm{h})$ & $\mathrm{T}\left({ }^{\circ} \mathrm{C}\right)$ & $\begin{array}{l}Y \\
(\%)^{b}\end{array}$ & $\begin{array}{l}\mathrm{Ee} \\
(\%)^{\mathrm{c}}\end{array}$ \\
\hline 1 & $\mathbf{A}(5 \%)$ & Toluene & 2 & $\mathrm{rt}$ & 70 & 43 \\
\hline 2 & B $(5 \%)$ & Toluene & 3 & $\mathrm{rt}$ & 54 & -48 \\
\hline 3 & $\mathrm{C}(5 \%)$ & Toluene & 7 & $\mathrm{rt}$ & 64 & -78 \\
\hline 4 & D $(5 \%)$ & Toluene & 6 & $\mathrm{rt}$ & 66 & -78 \\
\hline 5 & $\mathbf{E}(5 \%)$ & Toluene & 1.5 & $\mathrm{rt}$ & 86 & -46 \\
\hline 6 & $\mathbf{F}(5 \%)$ & Toluene & 1.5 & $\mathrm{rt}$ & 89 & -78 \\
\hline 7 & $\mathbf{G}(5 \%)$ & Toluene & 2 & $\mathrm{rt}$ & 86 & 46 \\
\hline 8 & $\mathbf{F}(5 \%)$ & $\mathrm{CH}_{2} \mathrm{Cl}_{2}$ & 1 & $\mathrm{rt}$ & 91 & -80 \\
\hline 9 & F $(5 \%)$ & $\mathrm{Et}_{2} \mathrm{O}$ & 2 & $\mathrm{rt}$ & 92 & -91 \\
\hline 10 & $\mathbf{F}(5 \%)$ & EtOAc & 2 & $\mathrm{rt}$ & 88 & -87 \\
\hline 11 & $\mathbf{F}(5 \%)$ & $i \mathrm{Pr}_{2} \mathrm{O}$ & 2 & $\mathrm{rt}$ & 94 & -87 \\
\hline 12 & F $(5 \%)$ & MTBE & 24 & $\mathrm{rt}$ & 30 & -91 \\
\hline 13 & $\mathbf{F}(5 \%)$ & $\mathrm{Et}_{2} \mathrm{O}$ & 2 & 0 & 90 & -97 \\
\hline 14 & $\mathbf{F}(2 \%)$ & $\mathrm{Et}_{2} \mathrm{O}$ & 24 & 0 & 80 & -98 \\
\hline 15 & $\mathbf{F}(1 \%)$ & $\mathrm{Et}_{2} \mathrm{O}$ & 24 & 0 & 35 & -96 \\
\hline
\end{tabular}

${ }^{\text {a) }}$ Reaction conditions: $0.100 \mathrm{mmol} \mathrm{1a}, 0.125 \mathrm{mmol} \mathrm{2}$, and catalyst in solvent $(1.0 \mathrm{~mL})$. ${ }^{\text {b) }}$ Isolated yield after column chromatography. ${ }^{c)}$ Enantiomeric excess determined by chiral HPLC.

Under the optimized conditions showed in entry 13, the substrate scope was investigated (Scheme 2). Different 1-naphthol ${ }^{[15]}$ substituted with electronwithdrawing and electron-donating groups were reacted with ethyl trifluoropyruvate ${ }^{[6]}$ (2). The presence of an electron-donating group such $\mathrm{MeO}$ at the position 4, decreased the yield, without compromising the enantiomeric excess (96\%), however groups such as $\mathrm{Cl}, \mathrm{Br}$ and $\mathrm{OAc}$ at this position gave good yields and excellent enantioselelectivities. Moreover, the reaction was studied with 1-naphthol substituted at 5-position, with good yields and excellent enantiomeric excesses. Remarkably, 1,5dihydroxynaphthalene reacted smoothly affording the corresponding chiral tertiary alcohol $\mathbf{3 g}$ with $70 \%$ 
yield and $97 \%$ ee ${ }^{[17]}$ Finally, our reaction protocol also allowed to use electron-rich phenols as nucleophiles. ${ }^{[18]} \quad$ Sesamol $^{[19]} \quad(\mathbf{4 a}), \quad 3,4-$ dimethoxyphenol (4b) and even phenols with only one electron-donating group (4c-4f) gave the corresponding chiral tertiary alcohols (5a-5f), although a decreased in the enantioselectivity was observed. Unfortunately, simple phenol is unreactive in our reaction conditions.
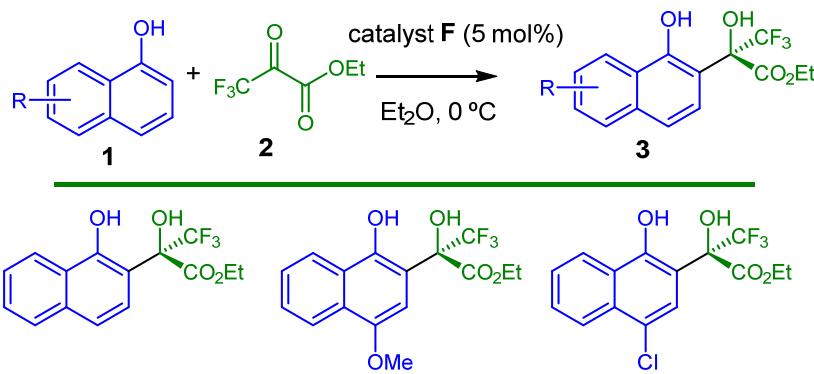

$\mathbf{3 a}^{\text {a }}{ }^{\text {90\% }}$ yield, $97 \%$ ee $\mathbf{3} \mathbf{b},{ }^{\text {a }} 49 \%$ yield, $96 \%$ ee $\mathbf{3} \mathbf{c}, 81 \%$ yield, $93 \%$ ee<smiles>CCOC(O)(c1cc(Br)c2ccccc2c1O)C(F)(F)F</smiles><smiles>CCOC(=O)C(O)(F)c1cc(OC(C)=O)c2ccccc2c1O</smiles><smiles>CCOC(O)(c1ccc2c(OC)cccc2c1O)C(F)(F)F</smiles>

3d, $63 \%$ yield, $99 \%$ ee

$3 e, 88 \%$ yield $97 \%$

$3 f^{\text {a }} 82 \%$ yield, $96 \%$ ee<smiles>CCOC(=O)C(O)(c1ccc2c(O)cccc2c1O)C(F)(F)F</smiles><smiles>CCOC(O)(c1ccc2c(Cl)cccc2c1O)C(F)(F)F</smiles><smiles>CCOC(O)(c1ccc2c(OC(C)=O)cccc2c1O)C(F)(F)F</smiles>

$\mathbf{3 g}^{\text {a }}{ }^{70 \%}$ yield, $97 \%$ ee $\mathbf{3} \mathbf{h},{ }^{\text {a }} 70 \%$ yield, $91 \%$ ee $\mathbf{3 i}, 72 \%$ yield, $97 \%$ ee<smiles>CCOC(=O)C(O)(c1cc2c(cc1O)OCO2)C(F)(F)F</smiles><smiles>CCOC(O)(c1cc(OC)c(OC)cc1O)C(F)(F)F</smiles><smiles>CCOC(O)(c1ccc(OC)cc1O)C(F)(F)F</smiles>

$\mathbf{5 a}, 70 \%$ yield, $77 \%$ ee $\quad \mathbf{5 b}, 68 \%$ yield, $86 \%$ ee $\quad \mathbf{5 c}, 81 \%$ yield, $87 \%$ ee<smiles>CCOC(O)(c1ccc(OCc2ccccc2)cc1O)C(F)(F)F</smiles><smiles>CCOC(=O)C(O)(c1ccc(OCC)cc1O)C(F)(F)F</smiles>

$\mathbf{5 d}, 64 \%$ yield, $88 \%$ ee 5 e, $50 \%$ yield, $71 \%$ ee<smiles>CCOC(O)(c1cc(Br)c(OC)cc1O)C(F)(F)F</smiles>

Scheme 2. Substrate scope for the enantioselective FriedelCrafts alkylation. Reaction conditions: $0.100 \mathrm{mmol} \mathrm{1,0.125}$ $\mathrm{mmol} \mathbf{2}$, and $\mathbf{F}(5 \mathrm{~mol} \%)$ in $\mathrm{Et}_{2} \mathrm{O}(1.0 \mathrm{~mL})$ at $0{ }^{\circ} \mathrm{C}$. Isolated yield after column chromatography. Enantiomeric excess determined by chiral HPLC. ${ }^{a}$ Compounds $3 \mathbf{a}, \mathbf{3 b}, \mathbf{3 f}, \mathbf{3 g}$ and $\mathbf{3 h}$, were isolated with a minor impurity that corresponds to the lactone.

The absolute configuration of product $\mathbf{3 c}$ was assigned as $(R)$ on the basis of an X-ray crystal structure analysis (Figure 2). ${ }^{[20]}$ The configuration of the rest of products were assigned on the assumption of a uniform mechanistic pathway.

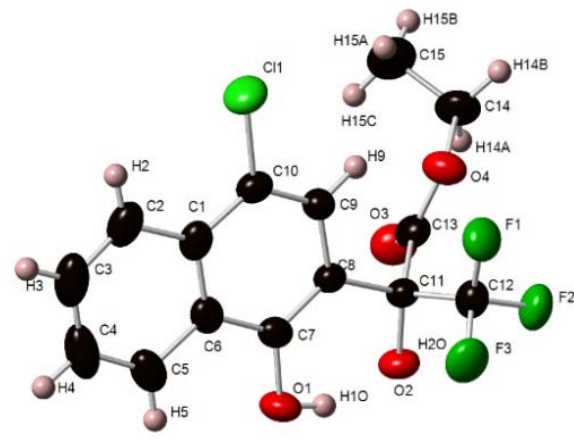

Figure 2. X-ray crystal structure of $\mathbf{3 c}$.

Treatment of product 3a with $p \mathrm{TsOH}$ in toluene at 70 ${ }^{\circ} \mathrm{C}$ afforded chiral lactone ${ }^{[21}{ }^{6} \mathbf{6}$ in $80 \%$ yield and preserving the enantiomeric purity of the compound (Scheme 3).<smiles>CCOC(O)(c1ccc2ccccc2c1O)C(F)(F)F</smiles>

$3 a$<smiles>O=C1Oc2c(ccc3ccccc23)[C@]1(O)C(F)(F)F</smiles>

6, $78 \%$ yield, $97 \%$ ee
Scheme 3. Lactonization of 3a.

In summary, we have developed a highly enantioselective addition of 1-naphthol derivatives to ethyl trifluoropyruvate employing a squaramide organocatalyst derived from quinine. The corresponding chiral tertiary alcohols bearing a trifluoromethyl group were obtained with good yields and excellent enantioselectivities. Furthermore the methodology was extended to the use of activated phenols, with good yields and enantiomeric excesses.

\section{Experimental Section}

\section{General Friedel-Crafts Procedure}

Naphthol 1 (0.100 mmol), ethyl trifluoropyruvate 2 (0.125 mmol) and squaramide F (2.4 mg, $0.050 \mathrm{mmol})$ were dissolved in $1.0 \mathrm{~mL}$ of ether and the mixture stirred at $0{ }^{\circ} \mathrm{C}$ until the reaction was complete (TLC). Finally, the reaction mixture was directly poured into the column chromatograpy, using hexane: $\mathrm{Et}_{2} \mathrm{O}(95: 5)$ as eluent to afford product 3 .

\section{Synthesis of (R)-3-hydroxy-3-(trifluoromethyl)} naphtho[1,2-b]furan-2(3H)-one (6)

A solution of 3a $(30 \mathrm{mg}, 0.095 \mathrm{mmol})$ and $p \mathrm{TsOH}(4 \mathrm{mg}$, $0.019 \mathrm{mmol})$ in toluene $(0.4 \mathrm{~mL})$ was stirred for $6 \mathrm{~h}$ at $70^{\circ} \mathrm{C}$. The corresponding reaction mixture was purified directly by flash chromatography on silica gel affording compound $\mathbf{6}$ (19.8 mg, 78\%).

\section{Acknowledgements}


Financial support from the MINECO (Gobierno de España and FEDER (EU)) (CTQ2013-47949-P) and from Generalitat Valenciana (ISIC2012/001) is gratefully acknowledged. M. M.-M. thanks the Universitat de València for a predoctoral grant. C. V. thanks MINECO for JdC contract. Access to NMR and MS facilities from the Servei central de support a la investigació experimental (SCSIE)-UV is also acknowledged.

\section{References}

[1] a) G. A. Olah, Friedel-Crafts Chemistry, Wiley, New York, 1973; b) R. M. Roberts, A. A. Khalaf, FriedelCrafts Alkylation Chemistry. A Century of Discovery, Dekker, New York, 1984.

[2] a) M. Bandini, A. Umani-Ronchi, Catalytic Asymmetric Friedel-Crafts Alkylations, Wiley-VCH, Weinheim, 2009; b) T. B. Poulsen, K. A. Jørgensen, Chem. Rev., 2008, 108, 2903; c) S.-L. You, Q. Cai, M. Zeng, Chem. Soc. Rev. 2009, 38, 2190.

[3] a) Bandini, M.; Eichlolzer, A. Angew. Chem. 2009, 121, 9786; Angew. Chem. Int. Ed., 2009, 48, 9608; b) G. Bartoli, G. Bencivenni, R. Dalpozzo, Chem. Rev. Soc. 2010, 39, 4449; c) R. Dalpozzo, Chem. Rev. Soc. 2015, 44,742 .

[4] a) S. Brandes, M. Bella, A. Kjærsgaard, K. A. Jørgensen, Angew. Chem. 2006, 118, 1165; Angew. Chem. Int. Ed. 2006, 45, 1147; b) S. Brandes, B. Niess, M. Bella, A. Prieto, J. Overgaard, K. A. Jørgensen, Chem. Eur. J. 2006, 12, 6039; c) T.-Y. Liu, H.-L. Cui, Q. Chai, J. Long, B.-J. Li, Y. Wu, L.-S. Ding, Y.-C. Chen, Chem. Commun. 2007, 2228; d) X.-S. Wang, G.-S. Yang, G. Zhao, Tetrahedron: Asymmetry 2008, 19, 709; e) X.-S. Wang, C.-W. Zheng, S.-L. Zhao, Z. Chai, G.; Zhao, G.S. Yang, Tetrahedron: Asymmetry 2008, 19, 2699; f) L. Hong, L. Wang, W. Sun, K. Wong, R. Wang, J. Org. Chem. 2009, 74, 6881; g) Y. Sohtome, B. Shin, N. Horitsugi, R. Takagi, K. Noguchi, K. Nagasawa, Angew. Chem. 2006, 118, 1550; Angew. Chem. Int. Ed. 2010, 49, 7299; h) X. Jiang, L. Wu, Y. Xing, L. Wang, S. Wang, Z. Chen, R. Wang, Chem. Commun. 2012, 446; i) E. Paradisi, P. Righi, A. Mazzanti, S. Ranieri, G. Bencivenni, Chem. Commun. 2012, 11178; j) L. Yu, X. Xie, S. Wu, R. Wang, W. He, D. Qin, Q. Liu, L. Jing, Tetrahedron Lett. 2013, 54, 3675; k) G. Liu, S. Zhang, H. Li, T. Zhang, W. Wang, Org. Lett 2011, 13, 828; 1) P. Chauhan, S. S. Chimni, Eur. J. Org. Chem. 2011, 1636; m) S. Takizawa, S. Hirata, K. Murai, H. Fujiokab, H. Sasai, Org. Biomol. Chem. 2014, 12, 5827; n) S. Takizawa, F. Arteaga-Arteaga, Y. Yoshida, J. Kodera, Y. Nagata, H. Sasai, Dalton Trans. 2013, 42, 11787; o) M. Montesinos-Magraner, C. Vila, R. Cantón, G. Blay, I. Fernández, M. C. Muñoz, J. R. Pedro, Angew. Chem. 2015, 127, 6418; Angew. Chem. Int. Ed. 2015, 54, 6320; p) Y. Sohtome, B. Shin, N. Horitsugi, K. Noguchi, K. Nagasawa, Chem. Asian J. 2011, 6, 2463; q) C. JaravaBarrera, F. Estaban, C. Navarro-Ranninger, A. Parra, J. Alemán, Chem. Commun. 2013, 49, 2001.

[5] G. Erker, A. A. H. van der Zeijden, Angew. Chem. 1990, 102, 543; Angew. Chem. Int. Ed. 1990, 29, 512.
[6] a) D. Wu, X. Zhang, Y. Xu, Y. Xue, J. Li, W. Wang, J. Zhu, Asian J. Org. Chem. 2014, 3, 480; b) J. Kaur, A. Kumar, S. S. Chimni, Tetrahedron Lett. 2014, 55, 2138.

[7] a) T. Hiyama, Organofluorine Compounds: Chemistry and Applications, Springer, Berlin, 2000; b) J. Wang, M. Sánchez-Roselló, J. L. Aceña, C. del Pozo, A. E. Sorochinsky, S. Fustero, V. A.. Soloshonok, H. Liu, Chem. Rev. 2014, 114, 2432; c) S. Purser, P. R. Moore, S. Swallow, V. Gouverneur, Chem. Soc. Rev. 2008, 37, 320; d) M. Shimizu, T. Hiyama, Angew. Chem. 2005, 117, 218; Angew. Chem. Int. Ed. 2004, 44, 214.

[8] a) K. Mikami, Y. Itoh, M. Yamanaka, Chem. Rev., 2004, 104, 1; b) J.-A. Ma, D. Cahard, Chem. Rev., 2004, 104, 6119; c) J. Nie, H.-C. Guo, D. Cahard, J.-A. Ma, Chem. Rev., 2011, 111, 455.

[9] a) S. Caron, N. M. Do, J. E. Sieser, P. Arpin, E. Vazquez, Org. Process Res. Dev. 2007, 11, 1015; b) N. Y. Rakhmanina, J. N. van den Anker, Expert Opin. Drug Metab. Toxicol. 2010, 6, 95.

[10] a) N. Gathergood, W. Zhuang, K. A. Jørgensen, J. Am. Chem. Soc. 2000, 122, 12517; b) W. Zhuang, N. Gathergood, R. G. Hazell, K. A. Jørgensen, J. Org. Chem. 2001, 66, 1009.

[11] For the use of indoles: a) B. Török, M. Abid, G. London, J. Esquibel, M. Török, S. C. Mhadgut, P. Yang, G. K. Surya Prakash, Angew. Chem. 2005, 117, 3146; Angew. Chem. Int. Ed. 2005, 44, 3086; b) M. P. A. Lyle, N. D. Draper, P. D. Wilson, Org. Lett. 2005, 7, 901; c) S. Nakamura, K. Hyodo, Y. Nakamura, N. Shibata, T. Toru, Adv. Synth. Cat. 2008, 350, 1443; d) Y. Hui, W. Chen, W. Eang, J. Jiang, Y. Cai, L. Lin, X. Liu, X. Feng, Adv. Synth. Cat. 2010, 352, 3174; e) C. Wolf, P. Zhang, Adv. Synth. Cat. 2011, 353, 760; f) X.-H. Xu, A. Kusuda, E. Tokunaga, N. Shibata, Green Chem. 2011, 13, 46; g) T. Sumiyoshi, K. Tojo, D. Urable, M. Tobe, Tetrahedron Asymmetry, 2011, 22, 153; h) G. Grach, A. Dinut, S. Marque, J. Marrot, R. Gil, D. Prim, Org. Biomol. Chem. 2011, 9, 497; i) X. Han, H.-B. Liu, C. Dong, Tetrahedron Asymmetry, 2012, 23, 1332; j) M. Rueping, T. Bootwicha, S. Kambutong, E. Suginomo, Chem. Asian. J. 2012, 7, 1195; k) X. Han, W. Ouyang, B. Liu, W. Wang, P. Tien, S. Wu, H.-B. Zhou, Org. Biomol. Chem. 2013, 11, 8463; 1) D. Carmona, P. Lamata, A. Sánchez, P. Pardo, R. Rodríguez, P. Ramírez, F. J. Lahoz, P. García-Orduña, L. A. Oro, Organometallics, 2014, 33, 4016; m) D. Carmona, M. P. Lamata, A. Sánchez, F. Viguri, R. Rodríguez, L. A. Oro, C. Liu, S. DiézGonzález, F. Maseras, Dalton Trans., 2014, 43, 11260. For the use of other heterocycles: n) K. Aikawa, Y. Asai, Y. Hioki, K. Mikami, Tetrahedron Asymmetry, 2014, 25, 1104. For the use of activated arenes: o) A. Corma, H. García, A. Moussaif, M. J. Sabater, R. Zniber, A Redouane, Chem. Commun. 2002, 1058; p) J.-L. Zhao, Y. Sui, Y.-L. Liu, D. Wang, Y.-J. Chen, Org. Lett. 2006, 8,6127 .

[12] For an organocatalytic example using simple phenols where the alkylation occurs at para position: J.-L. Zhao, L. Liu, C.-L. Gu, D. Wang, Y.-J. Chen, Tetrahedron Lett., 2008, 49, 1476. 
[13] a) G. Blay, I. Fernández, A. Monleón, J. R. Pedro, C. Vila, C. Org. Lett., 2009, 11, 441; b) G. Blay, I. Fernández, M. C. Muñoz, J. R. Pedro, C. Vila, Chem. Eur. J. 2010, 16, 9117; c) A. Sanz-Marco, A. GarcíaOrtiz, G. Blay, J. R. Pedro, Chem. Commun., 2014, 50, 2275; d) G. Blay, I. Fernández, A. Marco-Aleixandre, B. Monje, J. R. Pedro, R. Ruiz, Tetrahedron, 2002, 58, 8565.

[14] For a reviews of the use of squaramide catalyst: a) J. Alemán, A. Parra, H. Jiang, K. A. Jørgensen, Chem. Eur. J. 2011, 17, 6890; b) R. I. Storer, C. Aciro, L. H. Jones, Chem. Soc. Rev., 2011, 40, 2330; c) P. Chauhan, S. Mahajan, U. Kaya, D. Hack, D. Enders, Adv. Synth. Cat. 2015, 357, 253.

[15] 2-Naphthol was unreactive under the reaction conditions, probably due to steric hindrance.

[16] Methyl trifluoropyruvate was tested under the reaction conditions, but a mixture of $\alpha$-hydroxy- $\alpha-\mathrm{CF}_{3}$-esther and lactone (1:1.2 by ${ }^{19} \mathrm{~F}$ NMR) was observed, see supporting material for further details.

[17] Compounds 3a, 3b, 3f, $\mathbf{3 g}$ and $\mathbf{3 h}$ were isolated with a minor impurity that have been identified as the corresponding lactone. This lactone is formed during the course of the reaction, but we also observed the formation of the lactone during the isolation and purification processes of the product. This lactone has been fully characterized for the cyclization product of compound 3a, see Scheme 3.

[18] For an enantioselective additions of electron-rich phenols to isatines: a) J. Kaur, A. Kumar, S. S. Chimni, RSC Adv. 2014, 4, 62367; b) A. Kumar, J. Kaur, P. Chauhan, S. S. Chimni, Chem. Asian J. 2014, 9, 1605.

[19] a) A. M. Beekman, R. A. Barrow, J. Org. Chem. 2014, 79, 1017; b) W. J. Tsai, C. C. Shen, T. H. Tsai, L. C. Lin, J. Nat. Prod. 2014, 77, 125; c) P. Chauhan, S. S. Chimni, Tetrahedron Lett. 2013, 54, 4613; d) S. Bai, Y. Liao, L. Lin, W. Luo, X. Liu, X. Feng, J. Org. Chem. 2014, 79, 10662.

[20] CCDC 1405184 (3c) contains the supplementary crystallographic data for this paper. These data can be obtained free of charge from The Cambridge Crystallographic Data Centre via www.ccdc.cam.ac.uk/data_request/cif.

[21] For a racemic synthesis of lactone 6, see: V. I. Dyachenko, A. F. Kolomiets, A. V. Fokin, Izv. Akad. Nauk SSSR, Ser. Khim., 1987, 11, 2511. 


\section{COMMUNICATION}

Organocatalytic Enantioselective Friedel-Crafts Alkylation of 1-Naphthol Derivatives and Activated Phenols with Ethyl Trifluoropyruvate

Adv. Synth. Catal. Year, Volume, Page - Page

Marc Montesinos-Magraner, ${ }^{\mathrm{a}}$ Carlos Vila, ${ }^{\mathrm{a}}$ Gonzalo Blay, ${ }^{\mathrm{a}}$ Isabel Fernández, ${ }^{\mathrm{a}}$ M. Carmen Muñoz ${ }^{\mathrm{b}}$ and José R. Pedro ${ }^{\mathrm{a}, *}$

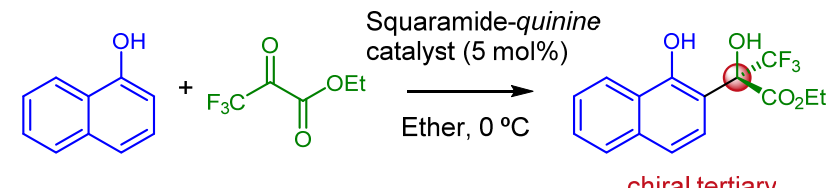

chiral tertiary trifluoromethyl alcohol $90 \%$ yield, $97 \%$ ee 